\title{
Lysate of Probiotic Lactobacillus plantarum K8 Modulate the Mucosal Inflammatory System in Dextran Sulfate Sodium-induced Colitic Rats
}

\author{
Young-Sook Ahn, Min Young Park, Jae-Ho Shin ${ }^{1}$, Ji Yeon Kim²,*, and Oran Kwon* \\ Department of Nutritional Science and Food Management, Ewha Womans University, Seoul 120-750, Korea \\ ${ }^{I}$ Department of Biomedical Laboratory Science, Eulji University, Seongnam 461-713, Korea \\ ${ }^{2}$ Department of Food Science and Technology, Seoul National University of Science and Technology, \\ Seoul 139-743, Korea
}

\begin{abstract}
Inflammatory bowel disease (IBD) is caused by dysregulation of colon mucosal immunity and mucosal epithelial barrier function. Recent studies have reported that lipoteichoic acid (LTA) from Lactobacillus plantarum K8 reduces excessive production of pro-inflammatory cytokine. In this study, we investigated the preventive effects of lysate of Lb. plantarum K8 in dextran sulfate sodium (DSS)-induced colitis. Male Sprague-Dawley rats were orally pretreated with lysate of Lb. plantarum K8 (low dose or high dose) or live Lb. plantarum K8 prior to the induction of colitis using 4\% DSS. Disease progression was monitored by assessment of disease activity index (DAI). Histological changes of colonic tissues were evaluated by hematoxylin and eosin (HE) staining. Tumor necrosis factor-alpha (TNF- $\alpha$ ), interleukin-6 (IL-6) levels were measured using enzyme-linked immunosorbent assay (ELISA). The colon mRNA expressions of TNF- $\alpha$, IL-6, and toll like receptor-2 (TLR2) were examined by quantitative real-time-transcription polymerase chain reaction (qPCR). Lysate of Lb. plantarum K8 suppressed colon shortening, edema, mucosal damage, and the loss of DSS-induced crypts. The groups that received lysate of $L b$. plantarum K8 exhibited significantly decreased levels of the pro-inflammatory cytokines TNF- $\alpha$ and IL-6 in the colon. Interestingly, colonic expression of toll like receptor- 2 mRNA in the high-dose lysate of $L$ b. plantarum K8 group increased significantly. Our study demonstrates the protective effects of oral lysate of Lb. plantarum K8 administration on DSS-induced colitis via the modulation of pro-inflammatory mediators of the mucosal immune system.
\end{abstract}

Keywords: colitis, lysate of Lactobacillus plantarum K8, pro-inflammatory cytokines, toll-like receptor 2

\section{Introduction}

Inflammatory bowel disease (IBD), which includes ulcerative colitis and Crohn's disease, is a severe, chronic, idiopathic autoimmune disease of the gastrointestinal tract (Podolsky, 2002). IBD is caused by the dysregulation of colon mucosal immunity and mucosal epithelial barrier function. Therefore, IBD patients may exhibit an abnormal expression of pro-inflammatory cytokines and other mediators that induce inflammatory activation of the colonic mucosal immune system (McGuckin et al., 2009; Neuman, 2007; Papadakis and Targan, 2000; Podolsky, 1991; Sartor, 2006). Current therapies for IBD in-

\footnotetext{
*Corresponding authors: Oran Kwon, Department of Nutritional Science and Food Management, Ewha Womans University, Seoul 120-750, Korea. Tel/Fax: +82-2-3277-6860, E-mail: orank @ewha.ac.kr; Ji Yeon Kim, Department of Food Science and Technology, Seoul National University of Science and Technology, Seoul 139-743, Korea. Tel/Fax: +82-2-970-6740, Email: jiyeonk@seoultech.ac.kr
}

clude pharmacological interventions, such as anti-inflammatory drugs and immunosuppressive antibiotics, but these therapies have limited benefits and significant side effects (Carvalho and Hyams, 2007; Triantafillidis et al., 2011). A more reliable strategy would be the total prevention of disease symptoms or non-pharmaceutical treatment, such as nutritional therapy with prebiotics and probiotics (Neuman and Nanau, 2012). Although probiotics are generally defined as live microorganisms, published evidence suggests that lysates or components isolated from probiotics function similarly to probiotics (Adams, 2010; Kataria et al., 2009; Zakostelska et al., 2011). Specifically, recent studies have reported that lipoteichoic acid (LTA), which is a cell wall component, obtained from $L b$. plantarum K8 induces lipopolysaccharide (LPS) tolerance and reduces the excessive production of pro-inflammatory cytokines and nitric oxide in the macrophage or monocytic cell lines (Kang et al., 2011; Kim et al., 2011; Ryu et al., 2009). Therefore, LTA from Lactobacillus plantarum K8 may control the homeostasis of intestinal infla- 
mmation during disease states, such as IBD. Based on these observations, we hypothesized that lysate of Lb. plantarum K8 containing LTA would also protect the histological mucosa from damage due to disruption by decreasing the levels of pro-inflammatory cytokines in colitisinduced rats. We also compared the effects of the cellular particles with those of live $L b$. plantarum K8.

\section{Materials and Methods}

\section{Preparation of Lb. plantarum lysate}

Lysate of $L b$. plantarum K8 containing LTA as $261 \mu \mathrm{g} /$ $10^{10} \mathrm{CFU}$ were provided by Sampyo Food Industry (Korea). Briefly, Lb. plantarum K8 (KCTC 10887BP, KCLB, Seoul, Korea) was grown in MRS broth at $37^{\circ} \mathrm{C}$ for $18 \mathrm{~h}$, the cells were harvested by centrifugation, and the cellular particles were produced using a high-pressure homogenizer (Avestin Inc., Canada). The contents of LTA was confirmed as previously described (Kang et al., 2011)

\section{Experimental design}

A total of 47 7-wk-old male Sprague-Dawley rats were adapted for $1 \mathrm{wk}$ and assigned into five groups via stratified randomization using body weight: the normal control group $(\mathrm{CON} / \mathrm{n}=10)$, a colitis control group ( $\mathrm{DSS} / \mathrm{n}=9)$, a group treated with live $L$ b. plantarum K8 $\left(1 \times 10^{9} \mathrm{CFU} / \mathrm{d}\right.$; $\mathrm{DLP} / \mathrm{n}=9$ ), and two groups treated with $L$ b. plantarum cellular particles in doses of $1 \times 0^{9} \mathrm{CFU} / \mathrm{d}$ or $1 \times 10^{10} \mathrm{CFU} /$ $\mathrm{d}(\mathrm{DDL} / \mathrm{n}=10$ and $\mathrm{DDH} / \mathrm{n}=9$, respectively). All groups were provided a diet of normal chow throughout the experimental period. On $0 \mathrm{~d}$ and continuing until $13 \mathrm{~d}$, the animals were orally gavaged once daily with either 500 $\mu \mathrm{L}$ of distilled water, live cells, or lysate of $L b$. plantarum K8. On $7 \mathrm{~d}, 4 \%$ DSS w/v (molecular weight, 36-50 kDa; MP Biomedicals, France) was substituted for drinking water in the DSS, DLP, DDL, and DDH groups to induce colitis, which was continued until $14 \mathrm{~d}$, when the animals were sacrificed. The CON group continued to drink distilled water throughout the entire experimental period. The Institutional Animal Care and Use Committee of Ewha Womans University approved the study protocol (approval \#2012-01-004).

\section{Assessment of colitic Disease Activity Index (DAI)}

The severity of colitis was assessed daily using a Disease Activity Index (DAI) based on the scoring system of Cooper et al. (Cooper et al., 1993), which scores body weight loss, stool consistency, and fecal bleeding on an increasing severity scale from 0 to 4 (Table 1). The DAI
Table 1. Disease Activity Index (DAI) scoring in DSS-induced colitis

\begin{tabular}{cccc}
\hline \hline Score & $\begin{array}{c}\text { Weight loss } \\
(\%)^{1}\end{array}$ & $\begin{array}{c}\text { Stool } \\
\text { consistency }^{2}\end{array}$ & $\begin{array}{c}\text { Fecal } \\
\text { bleeding }\end{array}$ \\
\hline 0 & $<0$ & Normal & Normal \\
1 & $1-5$ & - & - \\
2 & $6-10$ & Soft & Slightly bloody \\
3 & $11-20$ & Loose & Bloody \\
4 & $>20$ & Diarrhea & Severely bloody \\
\hline
\end{tabular}

${ }^{1}$ Weight loss was calculated [weight loss $(\%)=$ (Weight $_{\text {day } 21}-$ Weight $\left._{\text {day } 14}\right) /$ Weight $\left._{\text {day } 14} \times 100 \%\right]$ and scored.

${ }^{2}$ Stool consistency: Normal (well-formed pellets), Soft (do not adhere to the anus), Loose (pasty and semi-formed stools that adhere to the anus), Diarrhea (liquid stools that adhere to the anus).

was calculated as follows: (combined score for weight loss, stool consistency, and fecal bleeding)/3.

\section{Histological analysis}

The colon was excised from the cecum to the rectum at the end of the experimental period, and the middle colon $(1 \mathrm{~cm})$ was obtained for histopathological analysis. The colon samples were fixed immediately in a $10 \%$ formaldehyde solution, embedded in paraffin, cut into $5-\mu \mathrm{m}$ trans-verse sections, mounted on glass slides, deparaffinized, and stained with hematoxylin and eosin (H\&E) for histological assessment.

\section{Colonic inflammatory cytokine level assays}

The distal colon tissue samples were homogenized in $700 \mu \mathrm{L}$ lysis reagent (Sigma-Aldrich, USA) and $70 \mu \mathrm{L}$ protease inhibitor (Sigma-Aldrich). The homogenate was centrifuged at $20,000 \mathrm{~g}$ for $15 \mathrm{~min}$ at $4^{\circ} \mathrm{C}$ (Union 32R PLUS, Hanil, Korea), and the supernatants were stored at $-80^{\circ} \mathrm{C}$ until assays for the determination of cytokine levels were performed. The level of each cytokine was determined using a Duo Set enzyme-linked immunosorbent assay (ELISA) (R\&D Systems, USA) based on a sandwich ELISA, and the results are expressed as $\mathrm{pg} / \mathrm{mg}$ of protein in each sample. Briefly, 96-well plates were coated for 24 $\mathrm{h}$ at $4^{\circ} \mathrm{C}$ with a purified mouse anti-rat cytokine capture antibody. After washing three times with wash buffer, the wells were blocked with reagent diluent at room temperature for $1 \mathrm{~h}$ and washed three times with wash buffer. Recombinant rat cytokines were used as standards, and the colon samples were subsequently added. The plates were incubated at room temperature for $2 \mathrm{~h}$. After washing three times with wash buffer, a biotinylated goat antirat cytokine detection monoclonal antibody was added. BSA-PBS with $2 \%$ heat-inactivated normal goat serum was used for IL-6. The plates were incubated at room 
temperature for $2 \mathrm{~h}$ and washed three times with wash buffer. Streptavidin-conjugated horseradish peroxidase was added, and the plate was incubated at room temperature for $20 \mathrm{~min}$. After washing three times with wash buffer, a substrate solution was added, and the plate was incubated at room temperature for $20 \mathrm{~min}$. Finally, a stop solution was added to the plate, and the absorbance was read at $450 \mathrm{~nm}$ using a microplate reader (Eon Microplate Spectrophotometer, BioTek ${ }^{\circledR}$ Instruments, Inc., USA). Cytokine concentrations were determined using a standard curve and normalized to the amount of protein in the colon sample as measured using a BCA protein assay kit.

\section{Determination of colonic mRNA expression}

Total RNA was extracted from distal colonic samples using the TRIzol protocol (Invitrogen, USA). Singlestranded cDNA $(20 \mu \mathrm{g})$ was synthesized from total RNA using the High-Capacity cDNA Reverse Transcription Kit (Applied Biosystems, USA). The manufacturer's protocol for the PCR system was followed. The following TaqMan primer/probe sets were used to specifically detect the indicated target genes: TNF-a (Rn99999017_m1), IL6 (Rn99999011_m1), TLR-2 (Rn02133647_s1), and $\beta$ actin (Rn0067869_m1). The PCR reactions were performed in a 96-well Optical Reaction Plate (Applied Biosystems, USA), and $\beta$-actin, which was expressed at similar levels in all samples tested, was used as an endogenous control. Expression of the target genes was calibrated against the conditions found in the normal control animals.

\section{Statistical analysis}

Results are presented as means and standard errors (SE). The data were analyzed using Statistical Analysis Systems package version 9.2 (SAS Institute, USA). The differences between groups (CON, DSS, DLP, DDL, and DDH) were analyzed using one-way analysis of variance (ANOVA) with a post hoc Duncan's test. Statistical significance was indicated by $p<0.05$.

\section{Results}

\section{DAIs and colon lengths}

Lb. plantarum K8 or lysate of Lb. plantarum K8 did not overcome DSS-induced body weight suppression (data not shown), but the mean disease activity indexes (DAIs) in the DLP, DDL, and DDH groups were significantly lower than the DSS group at $4 \mathrm{~d}$ ( $p=0.0001$, Fig. 1A). However, no differences in the mean DAIs of all DSS-
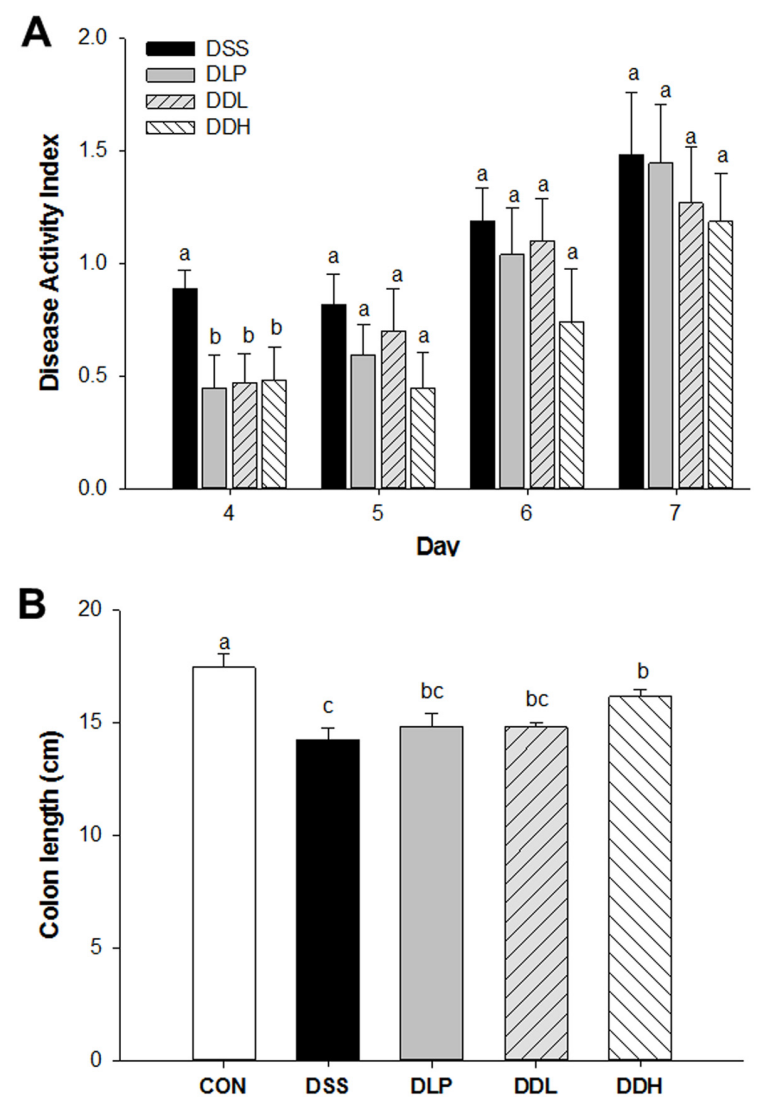

Fig. 1. Disease activity index (DAI) (A) and colon length (cm) (B) in DSS-induced colitic rats during the period of colitis induction. $\mathrm{CON}$ (Control, non-colitis induced, $\mathrm{n}=$ 10), DSS (colitis induced, $n=9$ ), DLP (colitis induced + $1 \times 10^{9} \mathrm{CFU} / \mathrm{d}$ live $L$ b. plantarum $\mathrm{K} 8, \mathrm{n}=9$ ), DDL (colitis induced $+1 \times 10^{9} \mathrm{CFU} / \mathrm{d}$ lysate of $L b$. plantarum $\mathrm{K} 8, \mathrm{n}=$ 10), DDH (colitis induced $+1 \times 10^{10} \mathrm{CFU} / \mathrm{d}$ lysate of $L b$. plantarum $\mathrm{K} 8, \mathrm{n}=9$ ). All values are expressed as means \pm SE. Statistical significance was determined using one-way ANOVA. Different letters at each day indicate significant differences at the $p<0.05$ level using Duncan's multiple range test.

treated groups were evident from $5 \mathrm{~d}$ because symptoms of DSS-induced colitis were exacerbated in all DSS-treated groups. Colon lengths are shown in Fig. 1B. The colon length of the DSS group (14.26 \pm 0.50$)$ was significantly shorter than the CON group (17.47 \pm 0.57$)$, whereas the DLP (14.86 \pm 0.57$)$ and DDL groups (14.80 \pm 0.23$)$ tended toward less colon shortening compared with the DSS group (Fig. 1B). Particularly, the colon length of the DDH group was significantly longer than the DSS group ( $p<$ 0.0001) among the experimental groups.

\section{Microscopic analysis of colonic tissue}

Microscopic colonic tissue architecture is shown in Fig. 2A. Severe destruction of epithelial cells, polymorphonu- 

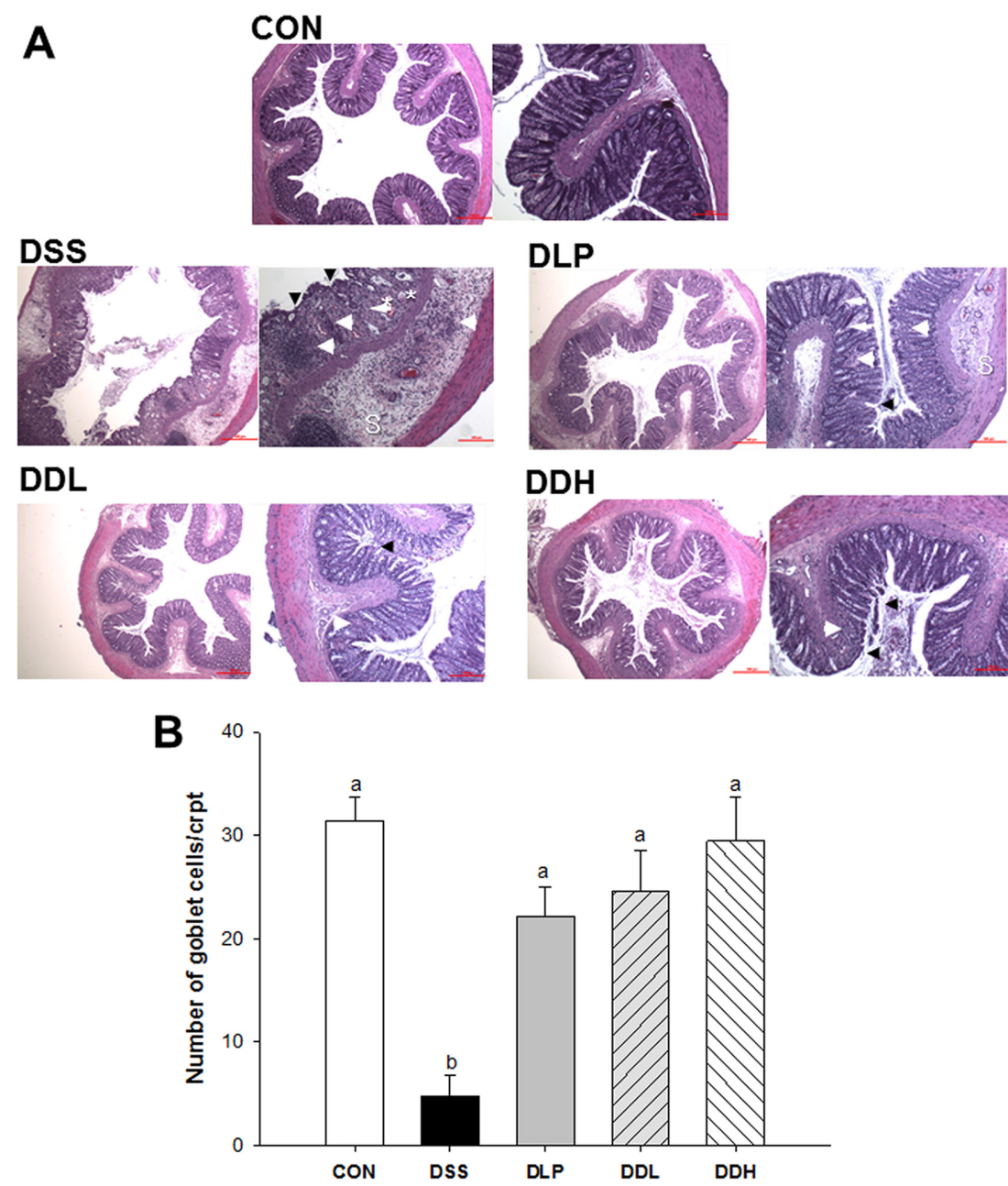

Fig. 2. Representative colon histopathology images of colons (A), and the number of goblet cells in colonic crypts (B) in DSSinduced colitic rats during the period of colitis induction. CON (Control, non-colitis induced, $\mathrm{n}=10$ ), DSS (colitis induced, $\mathrm{n}=$ 9), DLP (colitis induced $+1 \times 10^{9} \mathrm{CFU} / \mathrm{d}$ live $L b$. plantarum K8, $\mathrm{n}=9$ ), DDL (colitis induced $+1 \times 10^{9} \mathrm{CFU} / \mathrm{d}$ lysate of Lb. plantarum $\mathrm{K} 8, \mathrm{n}=10$ ), $\mathrm{DDH}$ (colitis induced $+1 \times 10^{10} \mathrm{CFU} / \mathrm{d}$ lysate of $L$ b. plantarum $\mathrm{K} 8, \mathrm{n}=9$ ). All values are expressed as means $\pm \mathrm{SE}$. Statistical significance was determined using one-way ANOVA. Different letters indicate significant differences at the $p<0.05$ level using Duncan's multiple range test. Glandular epithelium destruction (arrowhead), neutrophil infiltration (arrow), edema (sharp arrow), cryptic dilatation $\left(^{*}\right)$, or submucosal extension of inflammation (S). H\&E stain; bar, $100 \mu \mathrm{m}$ and $500 \mu \mathrm{m}$.

clear cell infiltration, mucous epithelial necrosis, and edema in the mucosa and submucosa were observed in the colon wall mucosa in all DSS groups. Inflammation, inflammatory cell infiltration, and mucosal edema were reduced in the DLP, DDL and DDH groups. The lysate of Lb. plantarum K8-treated groups also showed a dosedependent improvement. In particular, the DDH group appeared to be completely protected from damage, even more than the DDL or DLP groups, based on a decrease in edema and cell destruction compared with the DSS group. The mean numbers of goblet cells in crypts from three areas on each H\&E slide are shown in Fig. 2B. The number of goblet cells was significantly reduced in the DSS group compared to the DLP, DDL, and DDH groups $(p<0.0001)$, which was similar to that in the CON group.

\section{Pro-inflammatory cytokines in the colon}

The levels of cytokines secreted into the colon are shown in Fig 3A. Colonic TNF- $\alpha$ levels were significantly increased in the DSS group compared to the CON group. The DLP, DDL, and DDH groups had significantly lower colonic TNF- $\alpha$ levels compared to the DSS group (Fig. $3 \mathrm{~A}, p=0.0094)$. The levels of colonic IL-6 in the DSS group were also significantly increased compared to the CON group, whereas colonic IL-6 levels in the DDL and DDH groups were significantly decreased compared to the DSS group (Fig. 3A, $p=0.0370$ ). However, a tendency toward a decrease in colonic IL-6 levels was observed in 
A
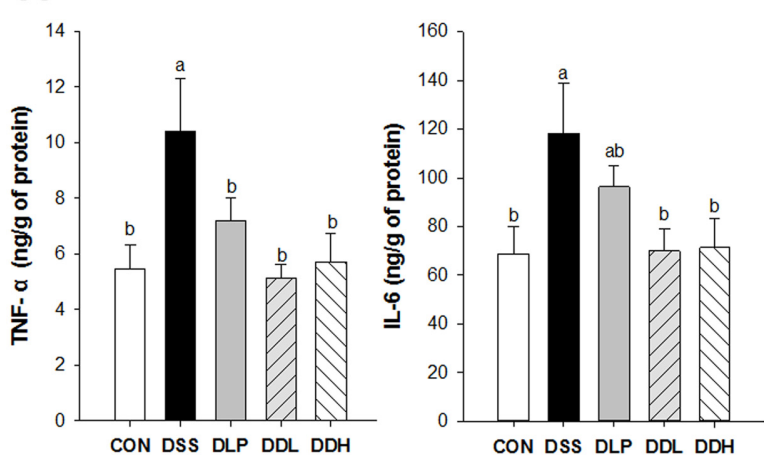

B
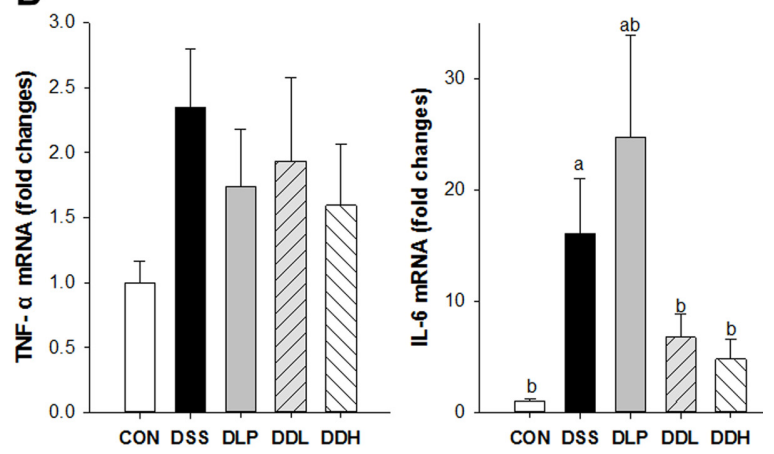

Fig. 3. Effect of live or lysate of $L b$. plantarum $\mathrm{K8}$ on colonic TNF- $\alpha$ and IL-6 levels (A) and their mRNA expression levels (B) in DSS-induced colitis rats. CON (Control, non-colitis induced, $n=10$ ), DSS (colitis induced, $n=$ 9), DLP (colitis induced $+1 \times 10^{9} \mathrm{CFU} / \mathrm{d}$ live $L$ b. plantarum $\mathrm{K} 8, \mathrm{n}=9$ ), DDL (colitis induced $+1 \times 10^{9} \mathrm{CFU} / \mathrm{d}$ lysate of Lb. plantarum $\mathrm{K} 8, \mathrm{n}=10$ ), DDH (colitis induced $+1 \times 10^{10}$ $\mathrm{CFU} / \mathrm{d}$ lysate of $L b$. plantarum $\mathrm{K} 8, \mathrm{n}=9$ ). All values are expressed as means \pm SE. Statistical significance was determined using one-way ANOVA. Different letters indicate significant differences at the $p<0.05$ level using Duncan's multiple range test.

the DLP group compared with the DSS group. The mRNA levels of TNF- $\alpha$ and IL- 6 are presented in Fig. 3B. The levels of TNF- $\alpha$ mRNA in the colon of the DSS group tended to increase compared with CON. TNF- $\alpha$ mRNA levels tended to be lower in the DLP, DDL, and DDH groups than the DSS group, but this difference was not significant between the experimental groups $(p=0.3418)$. IL-6 mRNA levels in the DSS group were significantly increased compared to the CON group. Furthermore, IL6 mRNA levels tended to be lower in the DDL and DDH groups than in the DSS group.

\section{Toll-like receptor 2 gene expression}

Colonic TLR-2 mRNA levels increased significantly in the DSS group compared to the CON group, whereas the levels of TLR-2 mRNA tended to decrease in the DLP

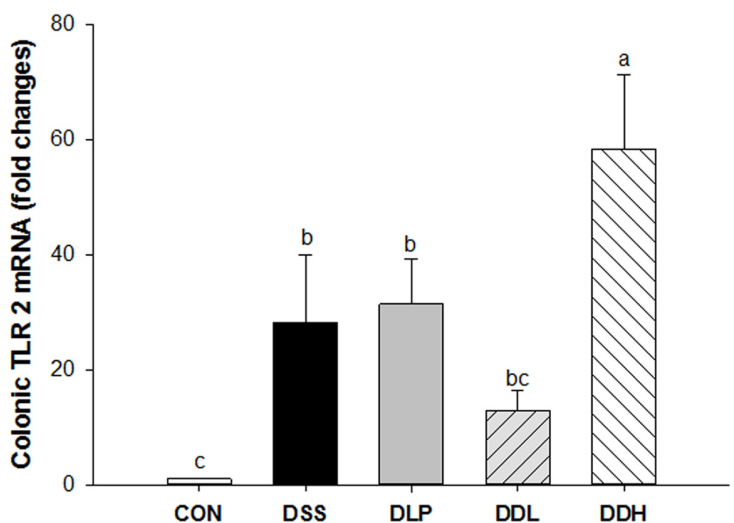

Fig. 4. Effect of live or lysate of $L b$. plantarum $\mathrm{K8}$ administration on colonic expression of toll-like receptor 2 (TLR-2) mRNA in DSS-induced colitic rats. CON (Control, non-colitis induced, $n=10$ ), DSS (colitis induced, $n=$ 9), DLP (colitis induced $+1 \times 10^{9} \mathrm{CFU} / \mathrm{d}$ live $L$ b. plantarum $\mathrm{K} 8, \mathrm{n}=9$ ), DDL (colitis induced $+1 \times 10^{9} \mathrm{CFU} / \mathrm{d}$ lysate of Lb. plantarum K8, $\mathrm{n}=10$ ), $\mathrm{DDH}$ (colitis induced $+1 \times 10^{10}$ CFU/d lysate of $L b$. plantarum $\mathrm{K} 8, \mathrm{n}=9$ ). All values are expressed as means \pm SE. Statistical significance was determined using one-way ANOVA. Different letters indicate significant differences at the $p<0.05$ level using Duncan's multiple range test.

and DDL groups compared with the DSS group (Fig. 4). Interestingly, TLR-2 mRNA levels increased significantly in the DDH group compared to the other groups ( $p=$ $0.0003)$.

\section{Discussion}

Lysate of $L b$. plantarum $\mathrm{K} 8$ and its constituent which is LTA exerted anti-inflammatory effects via the weak activation of TLR2 in previous studies in macrophage or monocytic cell lines (Kang et al., 2011; Kim et al., 2011; Ryu et al., 2009). Specifically, pretreatment with 10-100 $\mu \mathrm{g} / \mathrm{mL}$ of highly purified LTA isolated from $L b$. plantarum K8 reduced Gram-negative sepsis and induced tolerance upon LPS-stimulated monocyte activation via the inhibition of intracellular signal transduction and the reduction of pattern recognition receptors in human monocytic THP-1 cells (Kang et al., 2011; Kim et al., 2011). The present study confirmed that lysate of $L b$. plantarum $\mathrm{K} 8$ which contained LTA as $261 \mu \mathrm{g} / 10^{10} \mathrm{CFU}$ mitigate colitis pathophysiology by modulating the mucosal immune system in DSS-induced colitic. When the gastrointestinal fluid in the rats $(12.6 \mathrm{~mL})$ was considered (Mayhew and Carson, 1989), the administrated dosage (261 $\mu \mathrm{g} /$ $12.6 \mathrm{~mL}$ ) was consistent with the previous in vitro studies (Kang et al., 2011; Kim et al., 2011). In particular, a high 
dose of $L b$. plantarum K8 lysate significantly induced TLR-2 mRNA expression.

TLRs are responsible for microbial recognition, the induction of anti-microbial genes, and the control of the adaptive immune response (Li et al., 2010). Interestingly, besides TLR-4, which recognizes LPS, TLR-2 recognizes pathogenic and non-pathogenic peptidoglycan and LTA, which are principal components of Gram-positive bacteria, including the Lactobacillus genus (Han et al., 2003; Veckman et al., 2004). In a previous study, LTA obtained from $L b$. plantarum $\mathrm{K} 8$ acted as a TLR-2 activator and suppressed inflammation stimulated by LPS or Shigella flexneri peptidoglycan in the human monocytic THP-1 cells (Kim et al., 2011). Additionally, the present results show that colonic mRNA expression of TLR-2 in the DDH group was significantly increased compared with that in all of the experimental groups, whereas colonic TLR-2 mRNA expression in the DDL group tended to be lower than in the DSS and DLP groups. Several studies have reported a protective effect of TLR-2 on the intestinal epithelial barrier via the regulation of the expression of tight junction proteins (Cario, 2008; Cario et al., 2007; Rakoff-Nahoum et al., 2004; Yuki et al., 2011).

Pro-inflammatory mediators play central roles in the pathogenesis of IBD, including UC (Papadakis and Targan, 2000; Suzuki, 2012). The mucosal production of pro-inflammatory cytokines and immune cells are increased by enhanced intestinal permeability and the consequent immune cell infiltration. Therefore, the DSS-induced murine model and IBD patients show enhanced production of pro-inflammatory cytokines, such as TNF- $\alpha$ and IL- 6 (Neuman, 2007). A number of studies have reported that the administration of live or killed bacteria of the Lb. plantarum genus decreases colonic pro-inflammatory cytokines and/or increases anti-inflammatory cytokines (Chen et al., 2012; Fujiki et al., 2012; Geier et al., 2007; Mileti et al., 2009; Zhou et al., 2012). However, the physiological effects might be varied according to the strains. Therefore, we examined the levels and mRNA expression of colonic pro-inflammatory cytokines in the DSS-induced IBD rats treated with $L b$. plantarum K8 live cells or lysates. TNF- $\alpha$ is known to induce the inflammatory response and apoptosis in intestinal epithelial cells (Suzuki, 2012). In this study, the DLP, DDL, and DDH groups significantly decreased colonic TNF- $\alpha$ level and tended to decrease TNF- $\alpha$ mRNA expression compared with the DSS group. IL-6, which stimulate neutrophils and are associated with necrosis in the colon, are key mediators of IBD progression (Suzuki, 2012). In the present study, the DDL and DDH groups showed decreased colonic IL6 protein and mRNA expression levels compared to the DSS group. However, DLP administration decreased colonic pro-inflammatory cytokine levels, but no significant difference in colonic mRNA expression of pro-inflammatory cytokines was evident. Our results suggest that cellular particles are better anti-IBD nutritional supplements than live probiotics at the same doses because greater exposures of LTA might be exposed to the colonic mucosa.

\section{Conclusions}

Lysate of $L b$. plantarum $\mathrm{K} 8$ was found to protect against damage to the histological mucosa and disruption of the balance of the immune system induced by DSS colitis. Lysate of Lb. plantarum K8 decreased neutrophil infiltration of the colonic mucosa and inhibited the production of pro-inammatory cytokines, including TNF- $\alpha$ and IL-6 mRNA levels. Furthermore, DSS-induced colitis symptoms, such as weight loss, diarrhea, fecal bleeding, and shortened colon length, were reduced. In particular, a high dose of $L b$. plantarum K8 lysate may mediate the TLR-2 signaling that is associated with tight junctions. Therefore, we suggest that the improvement in colonic immune function in the high-dose $L b$. plantarum $\mathrm{K} 8$ lysate $\left(1 \times 10^{10} \mathrm{CFU} / \mathrm{d}\right)$-administered group may result from TLR-2-mediated signaling and/or immune system regulation via the induction of LPS tolerance stimulated by LTA as a low-grade inflammation. Further studies should be performed to elucidate TLR-2-mediated signaling by determining its epithelial barrier function, and human clinical trials are needed for nutritional treatments for gastrointestinal inflammatory conditions including IBD using $L b$. plantarum K8 lysate.

\section{Acknowledgements}

We thank the staff of Sampyo for the preparation of $L b$. plantarum K8. This research was supported by the Ministry of Science, ICT and Future Planning (Basic Science Research Program of the National Research Foundation of Korea Project No. 2013R1A1A1005859, NRF 2012 M3A9C4048761 and BK21 PLUS 22A20130012143).

\section{References}

1. Adams, C. A. (2010) The probiotic paradox: live and dead cells are biological response modifiers. Nutr. Res. Rev. 23, 37-46. 
2. Cario, E. (2008) Barrier-protective function of intestinal epithelial Toll-like receptor 2. Mucosal. Immunol. 1, S62-S66.

3. Cario, E., Gerken, G., and Podolsky, D. K. (2007) Toll-like receptor 2 controls mucosal inflammation by regulating epithelial barrier function. Gastroenterol. 132, 1359-1374.

4. Carvalho, R. and Hyams., J. S. (2007) Diagnosis and management of inflammatory bowel disease in children. Semin. Pediatr. Surg. 16, 164-171.

5. Chen, Y. P., Hsiao, P. J. Hong, W. S. Dai, T. Y., and Chen, M. J. (2012) Lactobacillus kefiranofaciens M1 isolated from milk kefir grains ameliorates experimental colitis in vitro and in vivo. J. Dairy Sci. 95, 63-74.

6. Cooper, H. S., Murthy, S. N., Shah, R. S., and Sedergran, D. J. (1993) Clinicopathologic study of dextran sulfate sodium experimental murine colitis. Lab. Invest. 69, 238-249.

7. Fujiki, T., Hirose, Y., Yamamoto, Y., and Murosaki, S. (2012) Enhanced immunomodulatory activity and stability in simulated digestive juices of Lactobacillus plantarum L-137 by heat treatment. Biosci. Biotechnol. Biochem. 76, 918-922.

8. Geier, M. S., Butler, R. N., Giffard, P. M., and Howarth, G. S. (2007) Lactobacillus fermentum BR11, a potential new probiotic, alleviates symptoms of colitis induced by dextran sulfate sodium (DSS) in rats. Int. J. Food Microbiol. 114, 267274.

9. Han, S. H., Kim, J. H., Martin, M., Michalek, S. M., and Nahm, M. H. (2003) Pneumococcal lipoteichoic acid (LTA) is not as potent as staphylococcal LTA in stimulating Toll-like receptor 2. Infect. Immun. 71, 5541-5548.

10. Kang, S. S., Ryu, Y. H., Baik, J. E., Yun, C. H., Lee, K., Chung, D. K., and Han, S. H. (2011) Lipoteichoic acid from Lactobacillus plantarum induces nitric oxide production in the presence of interferron-gamma in murine macrophages. Mol. Immunol. 48, 2170-2177.

11. Kataria, J., Li, N., Wynn, J. L., and Neu, J. (2009) Probiotic microbes: do they need to be alive to be beneficial? Nutr. Rev. 67, 546-550.

12. Kim, H. G., Lee, S. Y., Kim, N. R., Lee, H. Y., Ko, M. Y., Jung, B. J., Kim, C. M., Lee, J. M., Park, J. H., Han, S. H., and Chung, D. K. (2011) Lactobacillus plantarum lipoteichoic acid down-regulated Shigella flexneri peptidoglycan-induced inflammation. Mol. Immunol. 48, 382-391.

13. Li, X., Jiang, S., and Tapping, R. I. (2010) Toll-like receptor signaling in cell proliferation and survival. Cytokine. 49, 1-9.

14. Mayhew, T. M. and Carson, F. L. (1989) Mechanisms of adaptation in rat small intestine: regional differences in quantitative morphology during normal growth and experimental hypertrophy. J. Anat. 164, 189-200.

15. McGuckin, M. A., Eri, R., Simms, L. A., Florin, T. H., and Radford-Smith, G. (2009) Intestinal barrier dysfunction in inflammatory bowel diseases. Inflamm. Bowel. Dis. 15, 100113.

16. Mileti, E., Matteoli, G., Iliev, I. D., and Rescigno. M. (2009) Comparison of the immunomodulatory properties of three probiotic strains of Lactobacilli using complex culture systems: prediction for in vivo efficacy. PLoS One 4, e7056.

17. Neuman, M. G. (2007) Immune dysfunction in inflammatory bowel disease. Transl. Res. 149, 173-186.

18. Neuman, M. G. and Nanau, R. M. (2012) Inflammatory bowel disease: role of diet, microbiota, life style. Transl. Res. 160, 29-44.

19. Papadakis, K. A. and Targan, S. R. (2000) Role of cytokines in the pathogenesis of inflammatory bowel disease. Ann. Rev. Med. 51, 289-298.

20. Podolsky, D. K. (1991) Inflammatory bowel disease (1). $N$. Engl. J. Med. 325, 928-937.

21. Podolsky, D. K. (2002) Inflammatory bowel disease. N. Engl. J. Med. 347, 417-429.

22. Rakoff-Nahoum, S., Paglino, J., Eslami-Varzaneh, F., Edberg, S., and Medzhitov, R. (2004) Recognition of commensal microflora by toll-like receptors is required for intestinal homeostasis. Cell 118, 229-241.

23. Ryu, Y. H., Baik, J. E., Yang, J. S., Kang, S. S., Im, J., Yun, C. H., Kim, D. W., Lee, K., Chung, D. K., Ju, H. R., and Han, S. H. (2009) Differential immunostimulatory effects of Grampositive bacteria due to their lipoteichoic acids. Int. Immunopharmacol. 9, 127-133.

24. Sartor, R. B. (2006) Mechanisms of disease: pathogenesis of Crohn's disease and ulcerative colitis. Nat. Clin. Pract. Gastroenterol. Hepatol. 3, 390-407.

25. Suzuki, T. (2013) Regulation of intestinal epithelial permeability by tight junctions. Cell Mol. Life Sci. 70, 631-659.

26. Triantafillidis, J. K., Merikas, E., and Georgopoulos, F. (2011) Current and emerging drugs for the treatment of inflammatory bowel disease. Drug Des. Devel. Ther. 5, 185-210.

27. Veckman, V., Miettinen, M., Pirhonen, J., Sirén, J., Matikainen, S., and Julkunen, I. (2004) Streptococcus pyogenes and Lactobacillus rhamnosus differentially induce maturation and production of Th1-type cytokines and chemokines in human monocyte-derived dendritic cells. J. Leukoc. Biol. 75, 764-771.

28. Yuki, T., Yoshida, H., Akazawa, Y., Komiya, A., Sugiyama, Y., and Inoue, S. (2011) Activation of TLR2 enhances tight junction barrier in epidermal keratinocytes. J. Immunol. 187, 3230-3237.

29. Zakostelska, Z., Kverka, M., Klimesova, K., Rossmann, P., Mrazek, J., Kopecny, J., Hornova, M., Srutkova, D., Hudcovic, T., Ridl, J., and Tlaskalova-Hogenova, H. (2011) Lysate of probiotic Lactobacillus casei DN-114 001 ameliorates colitis by strengthening the gut barrier function and changing the gut microenvironment. PLoS One 6, e27961.

30. Zhou, Y. K., Qin, H. L., Zhang, M., Shen, T. Y., Chen, H. Q., Ma, Y. L., Chu, Z. X., Zhang, P., and Liu, Z. H. (2012) Effects of Lactobacillus plantarum on gut barrier function in experimental obstructive jaundice. World J. Gastroenterol. 18, 39773991 .

(Received 2014.9.26/Revised 2014.11.4/Accepted 2014.11.6) 